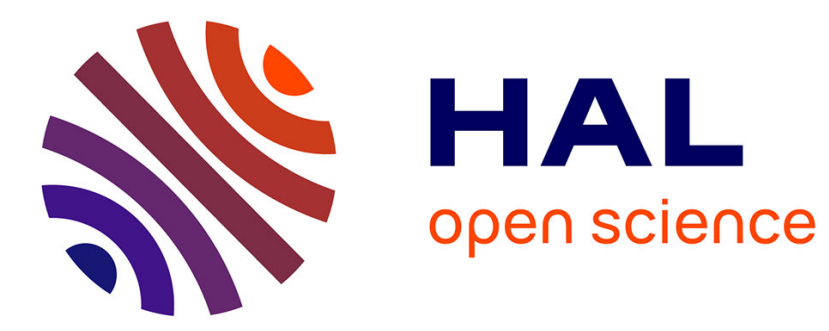

\title{
Experimental and theoretical study of processes leading to steady-state sound in annular thermoacoustic engines
}

Guillaume Penelet, Vitali Goussev, Pierrick Lotton, Michel Bruneau

\section{To cite this version:}

Guillaume Penelet, Vitali Goussev, Pierrick Lotton, Michel Bruneau. Experimental and theoretical study of processes leading to steady-state sound in annular thermoacoustic engines. Physical Review E : Statistical, Nonlinear, and Soft Matter Physics, 2005, 72 (1), 10.1103/physreve.72.016625 . hal02057449

HAL Id: hal-02057449

https://hal-univ-lemans.archives-ouvertes.fr/hal-02057449

Submitted on 5 Mar 2019

HAL is a multi-disciplinary open access archive for the deposit and dissemination of scientific research documents, whether they are published or not. The documents may come from teaching and research institutions in France or abroad, or from public or private research centers.
L'archive ouverte pluridisciplinaire HAL, est destinée au dépôt et à la diffusion de documents scientifiques de niveau recherche, publiés ou non, émanant des établissements d'enseignement et de recherche français ou étrangers, des laboratoires publics ou privés. 


\title{
Experimental and theoretical study of processes leading to steady-state sound in annular thermoacoustic engines
}

\author{
G. Penelet, ${ }^{1}$ V. Gusev, ${ }^{1,2}$ P. Lotton, ${ }^{1}$ and M. Bruneau ${ }^{1}$ \\ ${ }^{1}$ Laboratoire d'Acoustique de l'Université du Maine, UMR CNRS 6613, Avenue Olivier Messiaen, 72085 Le Mans Cedex 9, France \\ ${ }^{2}$ Laboratoire de Physique de l'Etat Condensé, UMR CNRS 6087, Avenue Olivier Messiaen, 72085 Le Mans Cedex 9, France
}

(Received 23 February 2005; published 28 July 2005)

\begin{abstract}
This paper gives a simplified analytical description of spontaneous generation and finite amplitude saturation of sound in annular thermoacoustic engines, and also provides comparison with experiments. The model includes the precise description of thermoacoustic amplification of sound (induced by interaction between an heterogeneously heated stack of solid plates and resonant gas oscillations), which accounts for the details of the temperature distribution in the whole thermoacoustic device (i.e., which does not only account for the mean temperature gradient along the stack). The saturation of the acoustic wave amplitude is described by taking into account both the reverse influence of high amplitude acoustic field on temperature field, and the dissipation of acoustic energy due to higher harmonics generation and minor losses (vortex generation). From the comparison between simulation results and experiments, it is demonstrated that the dynamical behavior observed in our experimental device is predominantly controlled by the effects of acoustic streaming and acoustically enhanced thermal conductivity tending not only to reduce the externally imposed temperature gradient along the stack, but also to change the shape of the temperature field.
\end{abstract}

DOI: 10.1103/PhysRevE.72.016625

PACS number(s): 43.25.+y

\section{INTRODUCTION}

Thermoacoustic prime movers, which involve the interaction between acoustic waves and temperature oscillations occurring near rigid walls inside acoustic boundary layers, are basically composed of a resonant acoustic tube and a stack of solid plates installed inside the tube to increase the thermal boundary layers effects. This stack can play the role of an acoustic driver when a strong temperature difference is applied between its ends by external thermal action: when the temperature gradient along the stack exceeds some critical value, the fluid particles in the thermoacoustic prime mover start to oscillate in the absence of any other external excitation (this is called thermoacoustic instability).

The physical principles of the thermoacoustic amplification process are nowadays fairly well understood, and linear thermoacoustic theory $[1,2]$ provides an effective tool for predicting the critical temperature gradient corresponding to the onset of the thermoacoustic instability in various thermoacoustic devices (this onset occurs when the thermoacoustic amplification produced in the stack exactly balances the linear thermal and viscous losses in the rest of the device). In particular, the analytical description of the thermoacoustic amplification process in annular thermoacoustic prime movers (see Fig. 1) without restriction on the stack length or on the shape of the temperature field has been proposed recently [3]. However, the well established foundations of thermoacoustics reach their limit when used to describe high amplitude thermoacoustic devices, and deeper understanding of nonlinear thermoacoustics requires more analytical investigations. In particular, it is important to characterize more precisely the physical mechanisms which lead to steady-state sound in thermoacoustic prime movers. Actually, because all the linear dissipation of sound is compensated by thermoacoustic amplification at the threshold of instability, these are only nonlinear effects which are able to stabilize the acoustic wave amplitude.

At the present time, a few nonlinear effects have been identified as potential explanations for the amplitude stabilization of the acoustic wave in thermoacoustic prime movers. The role of the cascade process of higher harmonics excitation has been studied both experimentally $[4,5]$ and theoretically [6-8], and the minor losses due to vortex generation at the edges of the stack and of the heat exchangers has been estimated $[9,10]$. Also, the processes involving the reverse influence of the acoustic waves on temperature distribution have been observed [11-16]. Among these mechanisms is the well-known effect of thermoacoustic heat transport from hot to cold ends of the stack induced by gas oscillations. This acoustically enhanced thermal conductivity [11] tends to re-

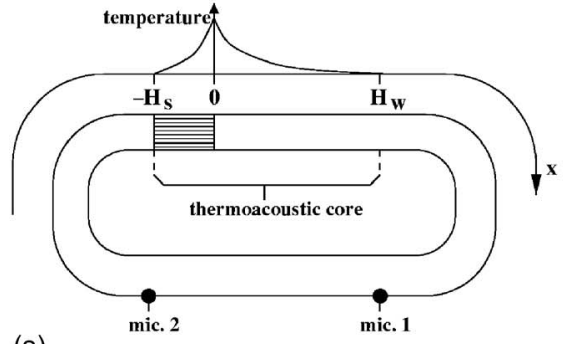

(a)

$1539-3755 / 2005 / 72(1) / 016625(13) / \$ 23.00$

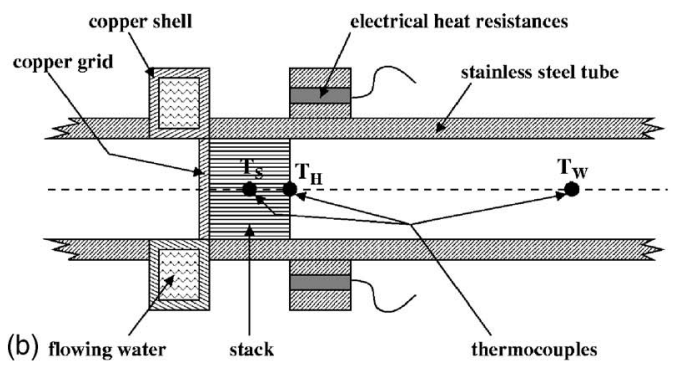

016625-1
FIG. 1. (a) Schematic diagram of the experimental apparatus. (b) Detailed representation of the thermoacoustic core. 
duce the externally imposed temperature gradient. Another mechanism which is expected to contribute to heat transport is the excitation of acoustic streaming. While the presence of acoustic streaming has been reported in several experimental devices [12-14], the role of the associated enthalpy flow on the thermoacoustic amplification process is poorly understood in thermoacoustic theory. Furthermore, the description of acoustic streaming needs to be reconsidered depending on whether the device is a standing wave prime mover or an annular ("traveling wave") thermoacoustic prime mover: in annular thermoacoustic prime movers the existence of a closed loop path results in the excitation of a mean unidirectional acoustic streaming carrying nonzero mass flow through each cross section of the resonator [17-19], while in standing wave devices the upward and downward streaming mass currents compensate necessarily each other in the resonator cross section.

While the direct numerical simulation of spontaneous generation and finite amplitude saturation of sound in thermoacoustic engines is limited by large computation times inherent to the multiscale complexity of the problem, the precise analytical description of thermoacoustic engine operation is a difficult task. We failed to find in the literature any (necessarily simplified) analytical description of the prime mover operation which accounts for all the above mentioned nonlinear processes and which agrees reasonably well with experimental results. In our opinion, this is partly due to the fact that the existing models consider a mean temperature gradient along the stack and do not allow for the temperature field to be other than linear in coordinate, whereas the actual temperature field generally has a nonlinear shape which may change significantly during the wave amplitude growth: for instance, the temperature profile in the "passive part" of the thermoacoustic core (i.e., in the region $0 \leqslant x \leqslant H_{\mathrm{w}}$ in Fig. 1) may change significantly as a consequence of forced convection due to acoustic streaming excitation. It is however demonstrated in Ref. [3] that the reverse influence of the temperature distribution on thermoacoustic amplification is significant because the temperature profile in the entire thermoacoustic core (including the "passive part") determines the spatial distribution of acoustic pressure and velocity amplitudes, and phase shift between pressure and velocity oscillations in the stack (i.e., in the "active" part of the thermoacoustic core where sound is amplified). Consequently, changes in the shape of the temperature field result in changes in the parameters controlling the thermoacoustic amplification process (or in other words, controlling the heat engine's cycle). This original result of Ref. [3] is an important one because it may explain the existing gap between experimental results and theory. Thus, the present study was initiated to reach a better understanding of the acoustic wave saturation in thermoacoustic devices, and to propose a model able to reproduce, at least qualitatively, the typical dynamic behaviors observed in experiments.

In this paper, experimental observations of the wave amplitude growth leading to steady-state sound in an annular thermoacoustic prime mover are first presented and analyzed in Sec. II. Then, in Sec. III, attention is focused on the analytical, time domain description of the interactions between acoustic and thermal fields due to acoustically enhanced ther- mal conductivity and acoustic streaming, using a one dimensional model to describe heat transfer in the thermoacoustic device. The model also accounts for the dissipaion of energy due to minor losses and higher harmonics generation. Section IV is devoted to the numerical finite difference resolution of the derived equations. The results obtained are compared with experiments: the dynamical behaviors observed in experiments are reproduced qualitatively, and also quantitatively in the simulations. Because it supplies a new understanding of fundamental processes which control the steadystate sound in thermoacoustic engines, this work contributes, in our opinion, to the progress in designing thermoacoustic engines so that they could become more efficient.

\section{EXPERIMENTAL RESULTS}

\section{A. Experimental apparatus}

A schematic diagram of the experimental apparatus is shown in Fig. 1. The torus-shaped stainless steel tube of inner radius $r_{i}=26.5 \mathrm{~mm}$ and length $L=2.24 \mathrm{~m}$ is filled with air at atmospheric pressure. The stack (length $H_{s}=5 \mathrm{~cm}$ ) is a ceramic porous material with square channels of cross section $0.9 \times 0.9 \mathrm{~mm}^{2}$. The cold heat exchanger is a copper circular shell set around the tube with flowing cooling water inside at room temperature $T_{\infty}$, and a copper wire mesh inside the tube (attached to the left side of stack). In order to impose a strong temperature gradient along the stack, eight electrical heat resistances connected to a thyristor unit are soldered in a stainless steel block set at the right side of the stack around the outer perimeter of the tube [Fig. 1(b)]. When the external thermal action on the system exceeds some critical value, the onset of the thermoacoustic instability results in the self-excitation of high amplitude acoustic waves oscillating at frequency $f \approx 153 \mathrm{~Hz}$, which corresponds approximately to the frequency of the first traveling wave mode of the annular resonator $(f \approx c / L$, where $c$ denotes the adiabatic sound velocity).

The instrumentation of the device is schematically shown in Fig. 1. Two piezoresistive microphones allow to measure acoustic pressure oscillations at two different positions along the resonator. The temperature measurement along the stack is carried out with five type $K$ thermocouples $(70 \mu \mathrm{m}$ in diameter) equally spaced along the median axis of the stack and glued with a ceramic putty. In order to investigate the possible inhomogeneity in the radial temperature distribution, two additional thermocouples are placed in the middle of the stack at $10 \mathrm{~mm}$ and $20 \mathrm{~mm}$ from the median axis, respectively. Additional type $K$ probes (1 $\mathrm{mm}$ in diameter) are also placed in the waveguide (see Fig. 1) to provide information on the heat transfer due to acoustic streaming. All sensors are linked to an A/D converter for signal processing.

\section{B. Experiments}

For each of the measurements presented in the following, the heating power supply is initially set to $Q_{0} \approx 100 \mathrm{~W}$ so that the device is just below the onset of the thermoacoustic instability. A small $\Delta Q$ increment is then sufficient for the 


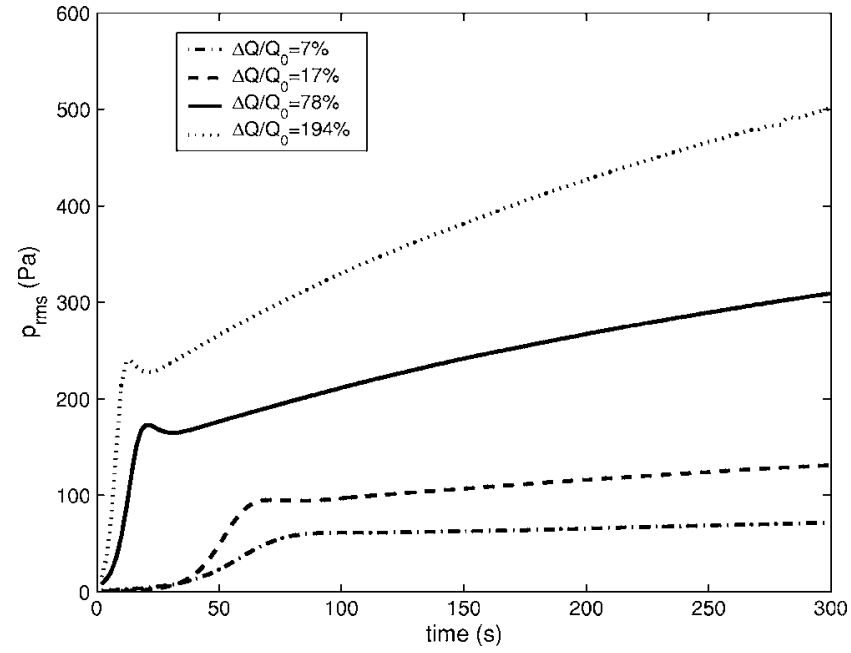

FIG. 2. Root mean square amplitude evolution of acoustic pressure $p_{\text {rms }}$ versus time, for various $\Delta Q$ increments of the external heating power supply above the critical value $Q_{0}$.

acoustic wave to be generated in the waveguide. Note that care was taken before each measurement to wait for temperature stabilization in the whole device.

Figure 2 presents the observed dynamics of the wave amplitude growth for various $\Delta Q$ increment on the external heating power supply above the critical value $Q_{0}$. For small $\Delta Q$ increments the onset of thermoacoustic instability results in the exponential growth of the acoustic wave amplitude, followed by a regime of stabilization. For large $\Delta Q$ increments, acoustic wave amplitude stabilization is followed by gradual amplification until the final stabilization. Figure 3 presents the evolution of the temperature distribution in the heterogeneously heated part of the device: the temperatures $T_{H}, T_{S}$, and $T_{W}$ [see Fig. 1(b)] are plotted versus time during
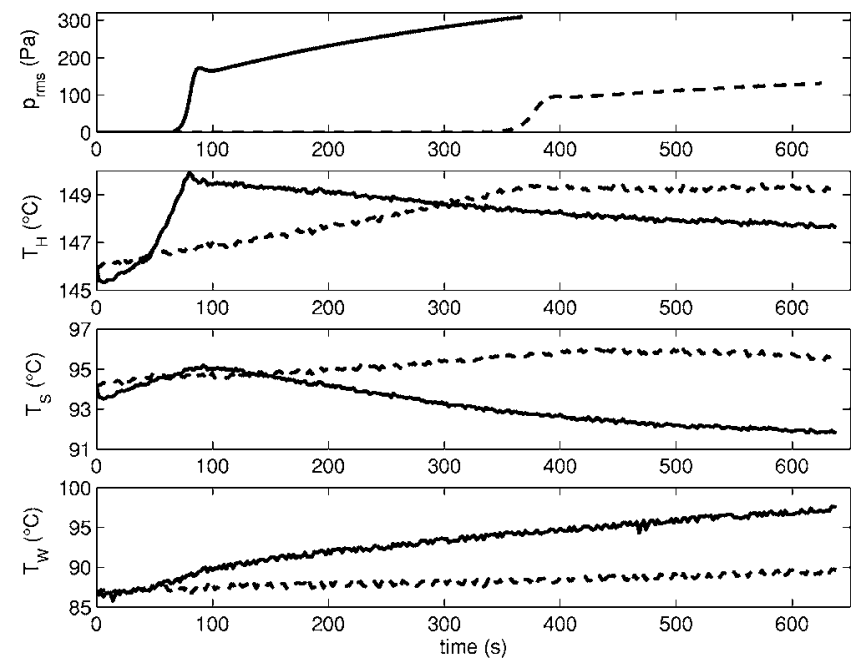

FIG. 3. Evolution of temperatures $T_{H}$ (at the hot end of the stack), $T_{S}$ (in the middle of the stack), and $T_{W}$ (at $12.5 \mathrm{~cm}$ right from the hot end of the stack in the waveguide) associated to different regimes of wave amplitude growth. Solid line: $\Delta Q / Q_{0}$ $=78 \%$; dashed line: $\Delta Q / Q_{0}=17 \%$. In both cases, $t=0$ corresponds to the time when the increment $\Delta Q$ is applied.



FIG. 4. Schematic representation of the thermoacoustic core, composed of three distinct homogeneous media.

two out of the four regimes plotted in Fig. 2. In both cases plotted respectively in dashed and solid lines in Fig. 3, $t=0$ corresponds to the time when the $\Delta Q$ increment is applied (this is not the case in Fig. 2). Two comments mainly arise from the results shown in Fig. 3. First of all, it appears in both cases that the onset of the acoustic wave is accompanied by significant acoustically induced changes in the temperature field; in particular, the observed gradual increase of $T_{W}$ may be attributed to the excitation of a unidirectional acoustic streaming carrying heat and mass flows in the clockwise direction $[13,18]$. Then, during the time dependent regime plotted in solid line in Fig. 3, it clearly appears that the first saturation of the acoustic wave amplitude (which occurs at time $t \approx 100 \mathrm{~s}$ ) corresponds to an acoustically induced break in the increase of $T_{H}$ (which is initially due to the external heating process); however in the subsequent evolution of acoustic pressure and temperatures $(t \geqslant 100 \mathrm{~s})$, the additional rise in the acoustic pressure amplitude is accompanied by a reduction of hot temperature $T_{H}$. This indicates that a simple temperature difference between the two ends of the stack is not a sufficient parameter to describe the thermoacoustic amplification process in that device, and confirms that the shape of the temperature field significantly impacts the thermoacoustic amplification process [3].

It must be mentioned here that the excitation of higher harmonics has been observed in the experiments described above, so that this effect may play a role in the saturation process. Also, the minor losses at the edges of the stack may be effective as well. Nevertheless, the experimental results clearly indicate that the reverse influence of the acoustic field on the temperature field needs to be described in an adequate theory, by including the heat transport by acoustic streaming and acoustically induced thermal conductivity in the heat transfer equations.

\section{SIMPLIFIED ANALYTICAL MODEL FOR THE TIME DEPENDENT REGIME}

In this section, the time dependent regime of the prime mover operation leading to steady-state sound is described by combining the equation describing the thermoacoustic amplification of sound with the equations describing, in a simplified one-dimensional approach, the heat transfer through the heterogeneously heated parts of the device (thermoacoustic core). As depicted in Fig. 4, the thermoacoustic 
TABLE I. Characteristic dimensions and thermophysical properties of materials used in the experimental device.

Thermophysical properties (at room temperature $T_{\infty}=294 \mathrm{~K}$ )

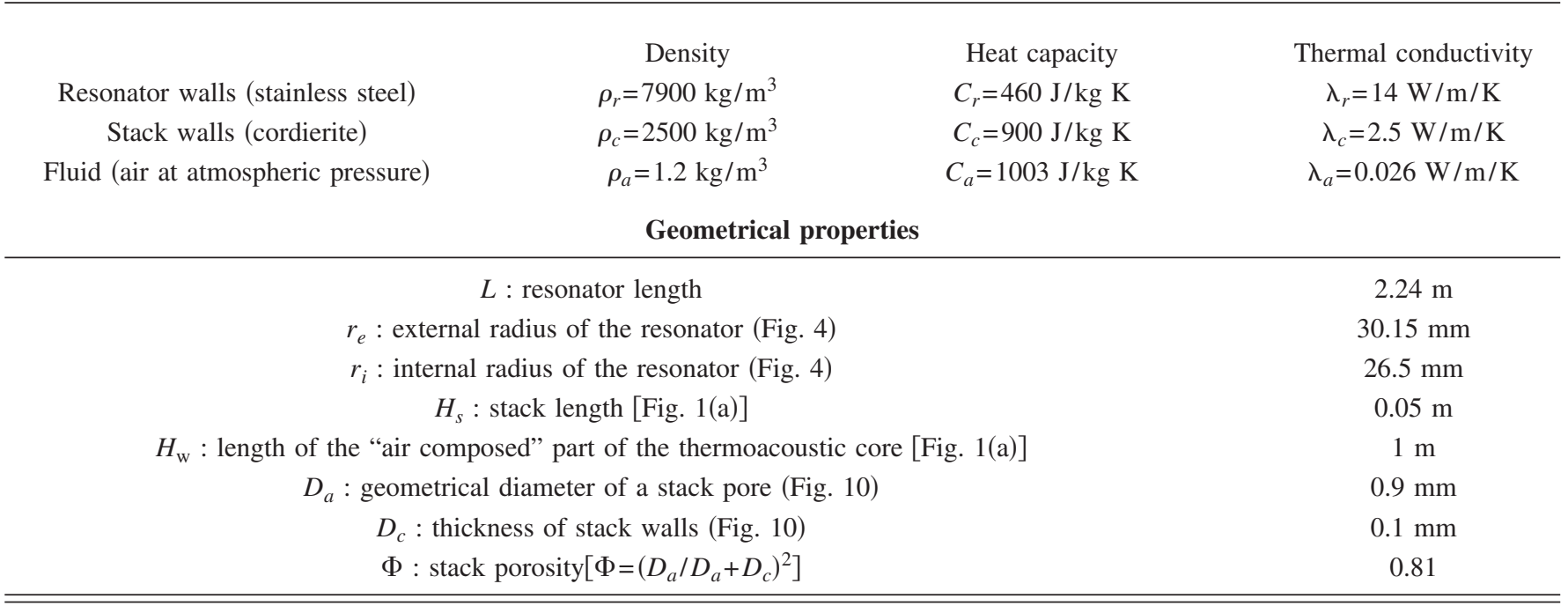

core is supposed to be composed of three homogeneous media: the resonator walls, the air in the heterogeneously heated part of the resonator, and the stack. In particular, the stack (composed both of air and ceramic) is considered as a homogeneous medium of thermal and physical properties:

$$
\begin{gathered}
\rho_{s} C_{s}=\rho_{a} C_{a} \Phi+\rho_{c} C_{c}(1-\Phi), \\
\lambda_{s}=\lambda_{a} \Phi+\lambda_{c}(1-\Phi),
\end{gathered}
$$

where subscripts $s, c, a$ refer to stack, ceramics, and air, respectively, and where $\Phi$ is the porosity of the stack. Table I reports the values for geometrical and thermophysical properties of materials which compose the experimental device [note that the temperature dependence of the thermal conductivity of air $\lambda_{a}$ is described using the empirical relation $\lambda_{a}(T)=\lambda_{a}\left(T_{\infty}\right) \times\left(T / T_{\infty}\right)^{0.73}[1]$, where $T_{\infty}$ stands for room temperature].

\section{A. Thermoacoustic amplification}

In a recent publication [3], the analytical description of the sound amplification in annular thermoacoustic prime movers has been proposed, without any restrictions either on the stack length or on the shape of the temperature field $T_{\mathrm{s}, \mathrm{w}}(x)$ in the thermoacoustic core. In the model, the amplification/attenuation of the acoustic wave in this closed loop device is represented by the thermoacoustic amplification coefficient $\alpha$ which can be calculated numerically, jointly with the corresponding frequency $f$ of acoustic oscillations, as a function of the sound scattering matrix coefficients of the thermoacoustic core. Here, using the analytical model presented in Ref. [3], the equation describing the evolution with time of the acoustic wave amplitude is written in the form of an ordinary differential equation as follows:

$$
d_{t} p_{1}=\frac{p_{1}}{\tau_{\text {ampl }}},
$$

where $d_{t}$ denotes time derivative and $p_{1}(t) \equiv p_{1}\left(x_{0}, t\right)$ stands for the root mean square amplitude of acoustic pressure oscillations at an arbitrary position $x_{0}$ along the device. The characteristic time $\tau_{\text {ampl }} \equiv \tau_{\text {ampl }}\left\{T_{\mathrm{s}, \mathrm{w}}(x, t)\right\}$ of thermoacoustic amplification depends on the temperature field spatial distribution in the thermoacoustic core. Simple calculations allow to express $\tau_{\text {ampl }}$ as a function of the thermoacoustic amplification coefficient $\alpha$ (defined in Ref. [3]) and corresponding acoustic frequency $f$ as follows:

$$
\tau_{\mathrm{ampl}}=\left(\alpha\left\{T_{\mathrm{s}, \mathrm{w}}\right\} \times f\left\{T_{\mathrm{s}, \mathrm{w}}\right\}\right)^{-1} .
$$

\section{B. Heat transfer in the thermoacoustic core}

In the simplified one-dimensional approach, the balance of heat transfer is drawn up in each of the three regions, i.e., the resonator walls (subscript " $r$ "), the stack (subscript " $s$ ") and the air in the nonhomogeneously heated part of the waveguide (subscript " $w$ "), as illustrated in Fig. 4. This results in a set of partial derivative equations which describe the coupling between the temperature fields in the resonator walls $\left[T_{r}(x, t)\right]$, in the stack $\left[T_{s}(x, t)\right]$ and in the waveguide $\left[T_{w}(x, t)\right]$ :

$$
\begin{gathered}
-H_{S}<x<0, \quad \rho_{r} C_{r} \partial_{t} T_{r}=\lambda_{r} \partial_{x x}^{2} T_{r}-\frac{2 \pi r_{i}}{\pi\left(r_{e}^{2}-r_{i}^{2}\right)} h^{r \leftrightarrow s}\left(T_{r}-T_{s}\right) \\
\quad-\frac{2 \pi r_{e}}{\pi\left(r_{e}^{2}-r_{i}^{2}\right)} h^{r \leftrightarrow \infty}\left(T_{r}-T_{\infty}\right), \\
0<x<H_{Q}, \quad \rho_{r} C_{r} \partial_{t} T_{r}=\lambda_{r} \partial_{x x}^{2} T_{r}-\frac{2 \pi r_{i}}{\pi\left(r_{e}^{2}-r_{i}^{2}\right)} h^{r \leftrightarrow w}\left(T_{r}-T_{w}\right) \\
+\frac{2 \pi r_{e}}{\pi\left(r_{e}^{2}-r_{i}^{2}\right)} \frac{Q(t)}{H_{Q}},
\end{gathered}
$$




$$
\begin{gathered}
H_{Q}<x<H_{W}, \quad \rho_{r} C_{r} \partial_{t} T_{r}=\lambda_{r} \partial_{x x}^{2} T_{r}-\frac{2 \pi r_{i}}{\pi\left(r_{e}^{2}-r_{i}^{2}\right)} \\
h^{r \leftrightarrow w}\left(T_{r}-T_{w}\right)-\frac{2 \pi r_{e}}{\pi\left(r_{e}^{2}-r_{i}^{2}\right)} h^{r \leftrightarrow \infty}\left(T_{r}-T_{\infty}\right), \\
-H_{S}<x<0, \quad \rho_{s} C_{s} \partial_{t} T_{s}+\rho_{s} C_{s} v_{s}(t) \partial_{x} T_{s}=\lambda_{s}(t) \partial_{x x}^{2} T_{s} \\
-\frac{2 \pi r_{i}}{\pi\left(r_{e}^{2}-r_{i}^{2}\right)} h^{r \leftrightarrow s}\left[T_{s}-T_{r}\right], \\
0<x<H_{W}, \quad \rho_{w} C_{w} \partial_{t} T_{w}+\rho_{w} C_{w} v_{w}(t) \partial_{x} T_{w}=\lambda_{w} \partial_{x x}^{2} T_{w} \\
-\frac{2 \pi r_{i}}{\pi\left(r_{e}^{2}-r_{i}^{2}\right)} h^{r \leftrightarrow w}\left[T_{w}-T_{r}\right],
\end{gathered}
$$

where $\partial_{t}$ and $\partial_{x}$ stand for partial time and space derivative, respectively. In Eq. (4) [Eq. (5), respectively], the influence of forced convection due to acoustic streaming on the temperature field is described by the terms $\rho_{s} C_{s} v_{s} \partial_{x} T_{s}$ (respectively $\rho_{w} C_{w} v_{w} \partial_{x} T_{w}$ ), where the dynamics of acoustic streaming establishment is controlled by the dynamics of acoustic pressure evolution $\left[v_{s, w}(t) \equiv v_{s, w}\left\{p_{1}(t)\right\}\right]$. The acoustic streaming velocity in the stack is an effective velocity which depends both on thermophysical properties of air and stack walls, and on stack porosity $\Phi$ (see Table I):

$$
v_{s}(t)=\frac{\Phi \rho_{a} C_{a}}{\rho_{s} C_{s}} \frac{v_{w}(t)}{\Phi} .
$$

Furthermore, in Eq. (4) the thermoacoustic heat pumping from hot to cold ends of the stack is described in the term $\lambda_{s} \partial_{x x}^{2}$ as an acoustically enhanced thermal conductivity $\lambda_{s}(t) \equiv \lambda_{s}\left\{p_{1}(t)\right\}$. In Eqs. (3b), (3c), and (5), the coupling between temperature fields $T_{w}(x, t)$ and $T_{r}(x, t)$ is described with the use of the phenomenological heat transfer coefficient $h^{r \leftrightarrow w}$ which accounts for the forced internal convection due to the excitation of acoustic streaming, while the coupling between temperature fields $T_{s}$ and $T_{r}$ [coefficient $h^{r \leftrightarrow s}$ in Eqs. (3a) and (4)] includes both radial heat diffusion in the stack walls and forced convection in the stack pores. Finally, the external heat leakage due to natural convection is described by the term $h^{r \leftrightarrow \infty}\left(T_{r}-T_{\infty}\right)$. In that simplified model, all of the coupling coefficients $h^{r \leftrightarrow w, r \leftrightarrow s, r \leftrightarrow \infty}$ are obtained from usual results of heat transfer theory [20] which give estimates for the Nusselt numbers associated to the device geometry and the nature of heat flux (natural/forced convection, internal/external convection, thermal radiation...). It must be mentioned however that the coefficients obtained are approximate values that account neither for the presence of an acoustic field (only the nonoscillatory-i.e., streaming induced-flow is considered) nor for the complicated radial distribution of streaming velocity (a unidirectional laminar flow is considered). The calculation details leading to the analytical expressions for $h^{r \leftrightarrow w, r \leftrightarrow s, r \leftrightarrow \infty}$ coefficients are reported in the Appendix.

In addition to Eqs. (3)-(5) describing heat transfer in the thermoacoustic core, the following boundary conditions are considered:

$$
\begin{gathered}
T_{r, s}\left(-H_{S}, t\right)=T_{\infty}, \\
T_{r, w}\left(H_{W}, t\right)=T_{\infty},
\end{gathered}
$$

which assume constant temperature $T_{\infty}$ at the ends of the thermoacoustic core, and

$$
\begin{gathered}
\partial_{x} T_{r}\left(0^{-}, t\right)=\partial_{x} T_{r}\left(0^{+}, t\right), \\
\partial_{x} T_{r}\left(H_{-}^{Q}, t\right)=\partial_{x} T_{r}\left(H_{+}^{Q}, t\right), \\
\lambda_{s}(t) \partial_{x} T_{s}\left(0^{-}, t\right)=\lambda_{w} \partial_{x} T_{w}\left(0^{+}, t\right)
\end{gathered}
$$

describing continuity of heat flux in the resonator walls and at the stack/air interface.

\section{Nonlinear saturation processes}

\section{Mean velocity of acoustic streaming}

In the one-dimensional approach of Eqs. (4) and (5), the effect of acoustic streaming on temperature field is simply represented by the forced convection terms $\rho_{s, w} C_{s, w} v_{s, w} \partial_{x} T_{s, w}$, where $v_{s, w}$ represent the mean acoustic streaming velocities through the resonator's internal cross section. The precise description of acoustic streaming in thermoacoustic engines is actually a much more complex problem, but it is assumed here, in this simplified one dimensional approach, that the main part of convective heat transfer is provided by that nonzero unidirectional mean mass flow.

The velocity of the mean unidirectional acoustic streaming in the stationary regime is estimated here from preliminary analytical and experimental works $[13,18]$. The analytical description of acoustic streaming in annular thermoacoustic prime movers has been proposed in Ref. [18]: it is demonstrated that the mean acoustic streaming velocity through the resonator's cross section is directed along $x$ axis that is clockwise [in accordance with Fig. 1(a)], and is proportional to the acoustic intensity. The dependence of the acoustic streaming velocity on the acoustic pressure in the present device has been also estimated experimentally in the stationary regime [13], using an indirect method which consists in comparing temperature distributions before and after the onset of thermoacoustic instability. Furthermore, the temporal dynamics of streaming establishment has been described analytically [21] in the simplified case of a pure traveling acoustic wave propagating in a nonheated annular resonator without stack: it is demonstrated ([21], Fig. 15) that the development of acoustic streaming in such a device can be modeled as follows:

$$
d_{t} v+\frac{v}{\tau}=\frac{v^{\max }}{\tau}
$$

where the acoustic streaming velocity in the stationary regime $v^{\max }$ is proportional to acoustic intensity. The characteristic time $\tau$ of streaming establishment depends on the regime of fluid/structure interaction as follows: 


$$
\tau \approx \frac{D^{2}}{\pi^{2} \nu}
$$

where $D$ denotes the resonator's internal diameter, and $\nu$ is the kinematic viscosity of fluid. In the present case, when a stack of solid plates (porosity $\Phi$ ) is introduced in the annular resonator (Fig. 1), the hydrodynamic pressure gradient $d_{x} P_{h}$ exists along the resonator axis and Eq. (8) must be replaced by

$$
\begin{gathered}
-H_{S} \leqslant x \leqslant 0, \quad d_{t} \frac{v}{\Phi}+\frac{1}{\tau_{s}} \frac{v}{\Phi}=\frac{1}{\tau_{s}} \frac{v^{\max }}{\Phi}-\frac{1}{\rho_{a}} d_{x} P_{h}, \\
0 \leqslant x \leqslant L-H_{S}, \quad d_{t} v+\frac{v}{\tau_{w}}=\frac{v^{\max }}{\tau_{w}}-\frac{1}{\rho_{a}} d_{x} P_{h},
\end{gathered}
$$

where the streaming velocity in the stack pores $v / \Phi$ is simply obtained from the velocity in the resonator $v$ using mass flow conservation and assuming incompressible flow $(v \ll c$, where $c$ denotes sound velocity). In Eqs. (10a) and (10b) the characteristic times $\tau_{s} \approx 2 \times 10^{-2} \mathrm{~s}$ and $\tau_{\mathrm{w}} \approx 20 \mathrm{~s}$ are obtained from Eq. (9) with geometrical properties of stack and resonator, respectively. Integrating over $x$ and adding together Eqs. (10a) and (10b), and accounting for $P_{h}(0)$ $=P_{h}(L)$ yields

$$
d_{t} v+\frac{v}{\tau_{v}}=\frac{v^{\max }}{\tau_{v}},
$$

where the global characteristic time of streaming establishment in the thermoacoustic prime mover $\tau_{v}$ is related to the characteristic times $\tau_{s}$ and $\tau_{w}$ by the following summation rule:

$$
\tau_{v}=\left(\frac{1}{L}\left[\frac{L-H_{S}}{\tau_{w}}+\frac{H_{S}}{\tau_{s}}\right]\right)^{-1} .
$$

Finally, since $v^{\max }$ is proportional to acoustic intensity, the equation describing the time evolution of the velocity of acoustic streaming in the resonator can be written as follows:

$$
d_{t} v_{w}+\frac{v_{w}}{\tau_{v}}=\frac{\Gamma_{v}}{\tau_{v}} p_{1}^{2}(t),
$$

where $\tau_{v} \approx 0.9 \mathrm{~s}$ is estimated from Eq. (12), while the coefficient $\Gamma_{v} \approx 4 \times 10^{-8} \mathrm{~m} / \mathrm{s}^{-1} \mathrm{~Pa}^{-2}$ describing the proportionality of the velocity of acoustic streaming with acoustic intensity is obtained from the experimental results presented in Ref. [13] (Fig. 5). Finally, the time evolution of the effective velocity in the stack region $v_{s}$ is obtained from Eqs. (6) and (13).

\section{Acoustically enhanced thermal conductivity}

In order to account for the thermoacoustic enthalpy flow in the stack, the thermal conductivity $\lambda_{s}$ is supposed to be time-dependent in Eqs. (4) and (7e). The variations with time of $\lambda_{s}$ can be written as follows:

$$
\lambda_{s}(t)=\lambda_{s_{0}}+\lambda_{s_{\mathrm{ac}}}(t),
$$

where $\lambda_{s_{0}}$ stands for the initial thermal conductivity $\left[\lambda_{s}\right.$ $\left.=\lambda_{a} \Phi+\lambda_{c}(1-\Phi)\right]$. The acoustically enhanced thermal conductivity $\lambda_{s_{a c}}$ is estimated from the well-known analytical expression for the thermoacoustic enthalpy flow $[1,2,7]$

$$
{\tilde{\lambda_{s}}}_{a c}=\frac{C_{a}}{2 \omega^{3} \rho_{a}} \frac{1-\sigma^{3 / 2}}{\left(1-\sigma^{2}\right) \sqrt{\sigma}}\left|\partial_{x} \tilde{p}\right|^{2} \operatorname{Im} f_{\nu},
$$

where $\tilde{p} \equiv \tilde{p}(x, \omega)$ is the complex amplitude of acoustic pressure oscillations $\left(p(x, t)=\operatorname{Re}\left[\tilde{p}(x, \omega) e^{-i \omega t}\right]\right), \sigma \approx 0.7$ is the Prandtl number of air, and where the well known function $f_{\nu}$ characterizes the efficiency of the viscous coupling of the acoustic field with the stack channel. This expression can be simplified here by analyzing the asymptotic cases of quasiadiabatic and quasi-isothermal regimes of stack/air interaction, respectively. Introducing the thermal and viscous acoustic boundary layers $\delta_{\kappa}=\sqrt{2 \kappa / \omega}$ and $\delta_{\nu}=\sqrt{2 \nu / \omega}$ (with $\kappa$ and $\nu$ denoting thermal diffusivity and kinematic viscosity of air, respectively), the asymptotic expression for acoustically induced thermal conductivity $\tilde{\lambda}_{s_{a c}}^{(\mathrm{QA})}$ in the quasiadiabatic regime $\left(\delta_{\kappa} / D_{a} \ll 1\right)$ is estimated [see Ref. [7], Eqs. (19),(23)] as follows:

$$
\tilde{\lambda}_{s_{a c}}^{(\mathrm{QA})} \approx \frac{C_{a}}{2 \omega \rho_{a} c^{2}} \frac{1-\sigma^{3 / 2}}{\left(1-\sigma^{2}\right) \sqrt{\sigma}} \frac{\delta_{\nu}}{2 D_{a}} \tilde{p}^{2},
$$

while its expression $\tilde{\lambda}_{s_{a c}}^{(\mathrm{QI})}$ in the case of a quasi-isothermal regime of thermoacoustic interaction $\left[\left(\delta_{\kappa} / D_{a}\right)^{2} \gg 1\right.$, see Ref. [7], Eqs. (41),(43)] as follows:

$$
\tilde{\lambda}_{s_{a c}}^{(\mathrm{QI})} \approx \frac{C_{a}}{2 \omega \rho_{a} c^{2}} \frac{1-\sigma^{3 / 2}}{\left(1-\sigma^{2}\right) \sqrt{\sigma}} 6\left(\frac{\delta_{\nu}}{D_{a}}\right)^{2} \tilde{p}^{2} .
$$

In both cases, the acoustically induced thermal conductivity is proportional to $\tilde{p}^{2}$. In the case of the experimental device considered in this paper, the thermoacoustic interaction in the stack channels is neither quasiadiabatic nor quasi-isothermal (in fact, $\delta_{\kappa} / D_{a} \approx 0.5$ ), but it is considered here for the sake of simplicity that the acoustically induced thermal conductivity is proportional to the square of mean acoustic pressure in the stack (as in the asymptotic cases):

$$
\lambda_{s}=\lambda_{s_{0}}+\Gamma_{\lambda} p_{1}^{2} \text {. }
$$

Consequently, the coefficient $\Gamma_{\lambda}$ can be estimated by interpolating its asymptotic expressions [Eqs. (16) and (17)] to the case corresponding to the experimental device $\left(\delta_{\kappa} / D_{a}\right.$ $\approx 0.5$ ). The following range

$$
6 \times 10^{-7} \leqslant \Gamma_{\lambda} \leqslant 2 \times 10^{-6}
$$

is finally obtained for $\Gamma_{\lambda}$.

\section{Higher harmonics generation and minor losses}

In order to make the analytical modelling of higher harmonics generation and minor losses tractable, a few assumptions need to be made. First of all, a traveling acoustic wave propagating in the $+x$ direction is considered, so that the time 
variations of the fundamental component of acoustic pressure $p_{1}(x, t)$ at an arbitrary position $x$ along the device can be written as follows:

$$
p_{1}(x, t) \approx p_{1}(t) \sin \left(\omega t-\frac{2 \pi x}{L}\right),
$$

where $\omega \approx 2 \pi c / L$ is the angular frequency of the fundamental acoustic mode. Secondly, the fluid is assumed to be weakly dissipative so that the acoustic velocity in the resonator is obtained from Euler's equation $\rho \partial_{t} v=-\partial_{x} p$ :

$$
v_{1}(x, t) \approx v_{1}(t) \sin \left(\omega t-\frac{2 \pi x}{L}\right),
$$

with

$$
v_{1}(t) \approx \frac{p_{1}(t)}{\rho_{a} c} .
$$

Higher harmonics generation. Because of classical nonlinear effects [22] the development of large amplitude acoustic waves in the present device results in the cascade process of higher harmonics generation. This change in the spectral content of the acoustic wave results in the increase of thermal and viscous dissipation near the resonator walls (proportional to the square root of acoustic frequency). Consequently, by hypothesizing that the acoustic wave saturation is only due to higher harmonics generation, the amplitude of acoustic waves would necessarily stabilize to a finite level so that the additional losses due to higher harmonics generation compensates for the thermoacoustic amplification of the fundamental mode. In the experiments, even the second harmonic has been found very small in comparison with the fundamental, so the cascade process of higher harmonics generation can be modelled by decomposing the temporal oscillations of the acoustic pressure wave just into its fundamental component $p_{1}(t)$ and its second harmonic $p_{2}(t)$

$$
p(t) \approx p_{1}(t) \sin (\omega \tau)+p_{2}(t) \sin (2 \omega \tau),
$$

where the delayed time $\tau=t-x / c$ characterizes the acoustic wave propagation, while the $t$ variations of acoustic pressure amplitude stand for slow variations in the time dependent regime of prime mover operation. This decomposition (22) is then introduced into the nonlinear equation of propagation without dispersion [22]

$$
\frac{\partial M}{\partial t}=\frac{\beta_{\mathrm{nl}}}{2} \frac{\partial M^{2}}{\partial \tau},
$$

where $\beta_{\mathrm{nl}}$ is the nonlinear parameter of fluid ( $\beta_{\mathrm{nl}}=1.2$ for air) and $M=p /\left(\rho_{a} c^{2}\right)$ is the acoustic Mach number, so that after identification of terms oscillating respectively at angular frequencies $\omega$ and $2 \omega$ the following set of equations is obtained:

$$
\frac{\partial M_{1}}{\partial t}=-\omega \frac{\beta_{\mathrm{nl}}}{2} M_{1} M_{2},
$$

$$
\frac{\partial M_{2}}{\partial t}=\omega \frac{\beta_{\mathrm{nl}}}{2} M_{1}^{2} .
$$

The simple physical sense of these coupled equations is that the second describes the generation of the second harmonic, while the first describes that the interaction of the fundamental frequency with the second harmonic leads to a leakage of energy from the fundamental wave. Finally, the analytical modeling of the saturation due to second harmonic generation is described by including in the model (24) the processes of amplification and absorption of sound waves:

$$
\begin{gathered}
\frac{\partial M_{1}}{\partial t}=-\omega \frac{\beta_{\mathrm{nl}}}{2} M_{1} M_{2}+\frac{M_{1}}{\tau_{\mathrm{ampl}}}, \\
\frac{\partial M_{2}}{\partial t}=\omega \frac{\beta_{\mathrm{nl}}}{2} M_{1}^{2}-\frac{M_{2}}{\tau_{\mathrm{att}}} .
\end{gathered}
$$

Here $M_{1} / \tau_{\text {ampl }}$ and $-M_{2} / \tau_{\text {att }}$ characterize the thermoacoustic amplification of fundamental mode [see Eq. (1)] and thermoviscous attenuation of second harmonic, respectively. The characteristic time $\tau_{\text {att }}$ is estimated in the present device by measuring (for various $\Delta Q / Q_{0}$ values) the stationary amplitudes $p_{1,2}(t \rightarrow \infty)$ and by introducing the obtained values in Eq. (25b) with $\partial_{t} M_{2}(t \rightarrow \infty)=0$ :

$$
\tau_{\text {att }}=\frac{2}{\omega \beta_{\mathrm{nl}}} \frac{M_{2}(t \rightarrow \infty)}{M_{1}^{2}(t \rightarrow \infty)} \approx 2.2 \times 10^{-2} \mathrm{~s} .
$$

It is important to remember here that a travelling wave propagating along $+x$ has been considered to model the cascade process of higher harmonics generation, whereas the actual acoustic field is much more complex $[3,12]$. Nevertheless, the acoustic field can be decomposed into its two counterpropagative components in the temperature homogeneous region (i.e., in the longest part of the resonator). Moreover, since the generation (with subsequent dissipation) of harmonics $3 \omega$ and higher has been neglected, there is no coupling in the nonlinear propagation of counterpropagative acoustic waves [23], so that the approach adopted is still valid in that case.

Minor losses. Minor losses refer to losses in flow energy which are physically associated with the formation of vortices, resulting here from changes in the form of flow cross section at the interface between the stack ends and resonator (Fig. 5). These additional losses are accompanied by an additional stress difference across the transition region, which is traditionally presented in terms of additional abrupt pressure variation $\Delta p$ in the region of abrupt change in tube cross section $[5,9,10,19]$. In the case of high Reynolds number steady flows, the pressure drop $\Delta p_{\text {out }}\left(x_{0}\right)=p_{\text {out }}\left(x_{2}\right)-p_{\text {out }}\left(x_{1}\right)$ associated for instance with an outgoing flow ("out" in Fig. 5 ) is proportional to the axial component of the flow velocity $V_{x}$ :

$$
\triangle p_{\text {out }}\left(x_{0}\right) \approx-\frac{1}{2} \rho_{a} K_{\text {out }} V_{x}^{2}\left(x_{0}\right) .
$$

Now, if a travelling acoustic wave [with associated velocity field $\left.v(t)=v_{1}(t) \sin (\omega \tau)\right]$ propagates in the closed loop reso- 


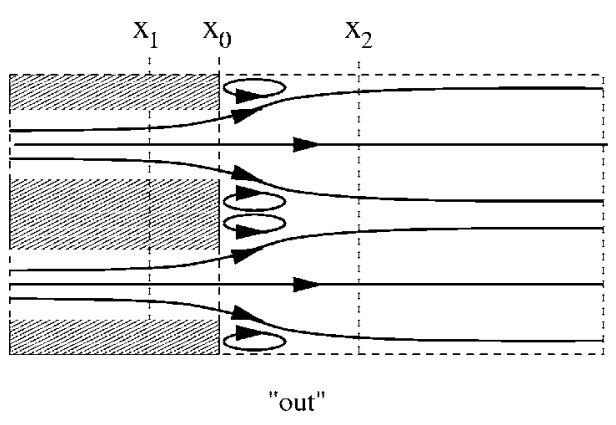

nator, the minor losses can be estimated by dissociating ingoing and outgoing flows:

$$
\begin{gathered}
\omega \tau_{0} \in[0, \pi], \Delta p\left(x_{0}, t\right) \approx-\frac{\rho_{a} K_{\mathrm{out}}}{2} v_{1}^{2}(t) \sin ^{2}\left(\omega \tau_{0}\right), \\
\omega \tau_{0} \in[\pi, 2 \pi], \Delta p\left(x_{0}, t\right) \approx+\frac{\rho_{a} K_{\mathrm{in}}}{2} v_{1}^{2}(t) \sin ^{2}\left(\omega \tau_{0}\right),
\end{gathered}
$$

with $\omega \tau_{0}=\omega t-2 \pi x_{0} / L$. Introducing the resonator's internal cross section $S_{w}=\pi r_{i}^{2}$ and the fluid portion of the stack's cross section $S_{s}=\Phi \times S_{w}$, it is possible to derive from Eq. (28) the expression of the minor losses induced decrease of acoustic power

$$
\begin{aligned}
\triangle \dot{E}_{\text {minor }}(t)= & \frac{\omega}{\pi} \int_{0}^{\pi} \Delta p_{\text {out }}(t) v_{1}(t) \sin \left(\omega \tau_{0}\right) S_{w} d\left(\omega \tau_{0}\right) \\
& +\frac{\omega}{\pi} \int_{\pi}^{2 \pi} \Delta p_{\text {in }}(t) v_{1}(t) \sin \left(\omega \tau_{0}\right) S_{s} d\left(\omega \tau_{0}\right) \\
& =-\frac{1}{2} \frac{4}{3 \pi} \rho_{a}\left(K_{\text {out }} S_{w}+K_{\text {in }} S_{s}\right) v_{1}^{3}(t)
\end{aligned}
$$

as a function of the amplitude $v_{1}(t)$ of acoustic velocity oscillations at the stack end. Then, using the expression of the total acoustic energy in the resonator (length $L$ )

$$
E_{\mathrm{tot}}(t)=\frac{S_{w}}{2} L \frac{p_{1}^{2}(t)}{\rho_{a} c^{2}},
$$

the decrease with time of total acoustic energy due to minor losses at the two stack ends

$$
\partial_{t} E_{\mathrm{tot}}=S_{w} L \frac{p_{1}}{\rho_{a} c^{2}} \partial_{t} p_{1}=2 \times \Delta \dot{E}_{\text {minor }}(t)
$$

is expressed as a decrease of the acoustic Mach number $M_{1}=p_{1} / \rho c^{2}$ which depends on $M_{1}$ itself and on a characteristic time $\tau_{\text {minor }}$ as follows:

$$
\partial_{t} M=-\frac{M^{2}}{\tau_{\text {minor }}},
$$

with
FIG. 5. Qualitative representation of vortices generation associated with an oscillatory flow at the stack ends.

This characteristic time $\tau_{\text {minor }}$ depends on the coefficients $K_{\text {in }}$ and $K_{\text {out }}$ which need to be estimated. Empirical relations (see Ref. [24], Chaps. 3 and 4) for the case of high Reynolds numbers $\left(\operatorname{Re} \geqslant 10^{4}\right)$ indicate the following expressions for minor losses coefficients: $K_{\text {out }} \approx\left(1-S_{s} / S_{w}\right)^{2}$ and $K_{\text {in }} \approx 0.5(1$ $\left.-S_{s} / S_{w}\right)^{3 / 4}$. Nevertheless, in the present experimental apparatus with a stack's pore diameter $D_{a} \approx 0.9 \mathrm{~mm}$, the associated Reynolds number $\operatorname{Re}=v_{1} D_{a} / \nu_{a} \approx p_{1} D_{a} /\left(\rho_{a} c \nu_{a}\right)$ is in the range $14 \leqslant \mathrm{Re} \leqslant 300$ when the acoustic pressure amplitude ranges from 100 to $2000 \mathrm{~Pa}$, so that the effect of fluid viscosity on the velocity distribution in one stack pore must be considered in the estimates of $K_{\text {in }}$ and $K_{\text {out }}$ [9,24]. Though we do not have here any empirical relations corresponding to the present device (with $S_{s} / S_{w}=0.81$ and $\mathrm{Re} \sim 10^{2}$ ), we use an order of magnitude estimate which has already been used in Ref. [19] (done on the basis of Ref. [24]), which consists of multiplying the above listed expression of $K_{\text {out }}$ and $K_{\text {in }}$ by factors 5 and 4 , respectively. Thus, reporting $K_{\text {out }} \approx 5 \times(1$ $\left.-S_{s} / S_{w}\right)^{2}$ and $K_{\text {in }} \approx 4 \times 0.5\left(1-S_{s} / S_{w}\right)^{3 / 4}$ in Eq. (33) yields $\tau_{\text {minor }} \approx 2.4 \times 10^{-2} \mathrm{~s}$.

Joint influence of minor losses and higher harmonics to wave saturation. Finally, from the simplified analytical models described in Secs. III C 3, the additional influence of minor losses and higher harmonics generation to acoustic wave saturation can be included in the model describing the interaction between acoustic and thermal fields. Indeed, Eq. (1) describing the time variations of the amplitude of acoustic pressure of fundamental mode must be replaced by the following set of equations:

$$
\begin{gathered}
\frac{\partial M_{1}}{\partial t}=\frac{M_{1}}{\tau_{\mathrm{ampl}}}-\omega \frac{\beta_{\mathrm{nl}}}{2} M_{1} M_{2}-\frac{M_{1}^{2}}{\tau_{\text {minor }}}, \\
\frac{\partial M_{2}}{\partial t}=\omega \frac{\beta_{\mathrm{nl}}}{2} M_{1}^{2}-\frac{M_{2}}{\tau_{\mathrm{att}}},
\end{gathered}
$$

which is derived from Eqs. (25) and (32), with $M_{1,2}$ $=p_{1,2} /\left(\rho c^{2}\right)$, and assuming that the main contribution to minor losses is that from the fundamental acoustic mode. 


\section{NUMERICAL SIMULATIONS}

\section{A. Prediction of wave amplitude dynamics, comparison with experiments}

In Sec. III, all the equations describing the prime mover operation from the onset of the thermoacoustic instability to its saturation due to nonlinear effects have been derived. Now, the problem is to simultaneously solve a set of partial differential equations describing the evolution with time of the temperature fields coupled to ordinary differential equations describing the evolution with time of both acoustic pressure amplitude and acoustic streaming velocity:

$$
\begin{gathered}
\partial_{t} T_{r}=F_{r}\left(t, T_{r}, T_{s}, T_{w}, \ldots\right), \quad[\text { from Eq . (3)], } \\
\partial_{t} T_{s}=F_{s}\left(t, T_{r}, T_{s}, v_{s}, p_{1}, \ldots\right), \quad[\text { from Eqs . (4), (18)], } \\
\partial_{t} T_{w}=F_{w}\left(t, T_{r}, T_{w}, v_{w}, p_{1}, \ldots\right), \quad[\text { from Eq . (5)], } \\
\partial_{t} v_{s, w}=F_{v}\left(t, p_{1}, \ldots\right), \quad[\text { from Eqs . (6), (13)], } \\
\partial_{t} p_{1,2}=F_{p}\left(t, T_{s}, T_{w}, p_{1}, p_{2}, \ldots\right) \quad[\text { from Eqs . (34), (2)]. }
\end{gathered}
$$

The solution is derived numerically, using finite difference methods: partial differential equations are solved using the Cranck-Nicholson scheme [25] while ordinary differential equations are solved with the classical Runge-Kutta method (order 4). In the following, the way used to compute the time dependent regime is presented.

First of all, the acoustic pressure is set to zero in the whole device, and the external heating power is set to a fixed value $Q_{0}$ in order for the system to be just below the onset of thermoacoustic instability after computing the associated steady thermal field $T_{r, s, w}(x)$. This condition is satisfied when the thermoacoustic amplification coefficient $\alpha$ calculated from the temperature distribution $T_{s, w}(x)$ inside the thermoacoustic core (using the method presented in Ref. [3]) is close to-but less than-zero (for instance $\alpha\left\{T_{s, w}\right\} \approx-10^{-5}$ ).

Then, at time $t=0$, the amplitude of acoustic pressure $p_{1}$ is set to a small value (e.g., $p_{1} \approx 10^{-2} \mathrm{~Pa}$ ), and the external heating is incremented to $Q_{0}+\Delta Q$ (the initial temperature field being the one computed previously).

The temperature field $T_{r, s, w}(x, t+\Delta t)$ and the corresponding characteristic time $\tau_{\text {ampl }}(t+\Delta t)$ of thermoacoustic amplification are computed, and then the values for other variables $\left(p_{1,2}, v_{s, w}, \lambda_{s}\right)$ are obtained for the time $t+\Delta t$. This step by step operation is repeated until the stabilization of the acoustic pressure amplitude.

\section{B. Results}

In the simulation results presented below, the numerical values of geometrical and thermophysical parameters are those listed in Table I, and the estimated values of the parameters controlling the nonlinear saturation processes are listed in Table II.

Concerning the comparison of simulation results with experiments, a point that has been deliberately hidden until now needs to be made clear: this point concerns the spatial
TABLE II. Estimated values of parameters controlling the nonlinear saturation processes.

\begin{tabular}{cc}
\hline \hline$\Gamma_{v}$ & $4 \times 10^{-8} \mathrm{~m} / \mathrm{s}^{-1} \mathrm{~Pa}^{-2}$ \\
$\tau_{v}$ & $0.9 \mathrm{~s}$ \\
$\Gamma_{\lambda}$ & $2 \times 10^{-6} \mathrm{~W} / \mathrm{m}^{-1} \mathrm{~K}^{-1} \mathrm{~Pa}^{-2}$ \\
$\tau_{\text {att }}$ & $2.2 \times 10^{-2} \mathrm{~s}$ \\
$\tau_{\text {minor }}$ & $2.4 \times 10^{-2} \mathrm{~s}$ \\
\hline \hline
\end{tabular}

distribution of the acoustic field in the whole device. To be precise, it must be mentioned, first of all, that in the model (Sec. III) the amplitude of acoustic pressure $p_{1}$ refers necessarily to acoustic pressure in the stack, because both acoustically enhanced conductivity and minor losses depend on acoustic pressure in the stack. Secondly, the experimental results presented in Sec. II correspond to measurements of acoustic pressure with the microphone "mic. 1" (see Fig. 1), that is approximately 1 metre away from the hot stack end. The distribution of the acoustic field in annular thermoacoustic prime movers has been studied both experimentally [12] and theoretically [3], and in both cases the results show that for a resonator of length $L$, the spatial distribution of acoustic pressure at fundamental frequency is $L / 2$ periodic. Consequently, the amplitude of acoustic pressure measured at $1 \mathrm{~m}$ $\approx L / 2$ (with $L=2.24 \mathrm{~m}$ ) from the stack is approximately the amplitude of acoustic pressure in the stack, and the simulation results are compared directly with experimental results of Fig. 2.

In the light of the experimental results presented in Sec. II, which indicate that the processes involving the reverse influence of the acoustic field on the temperature field may play a predominant role in the saturation of the acoustic wave, it seems reasonable here first to simulate time dependent regimes when the only effects of acoustic streaming and acoustically induced conductivity are included. Figure 6 presents such simulation results of the prime mover operation,

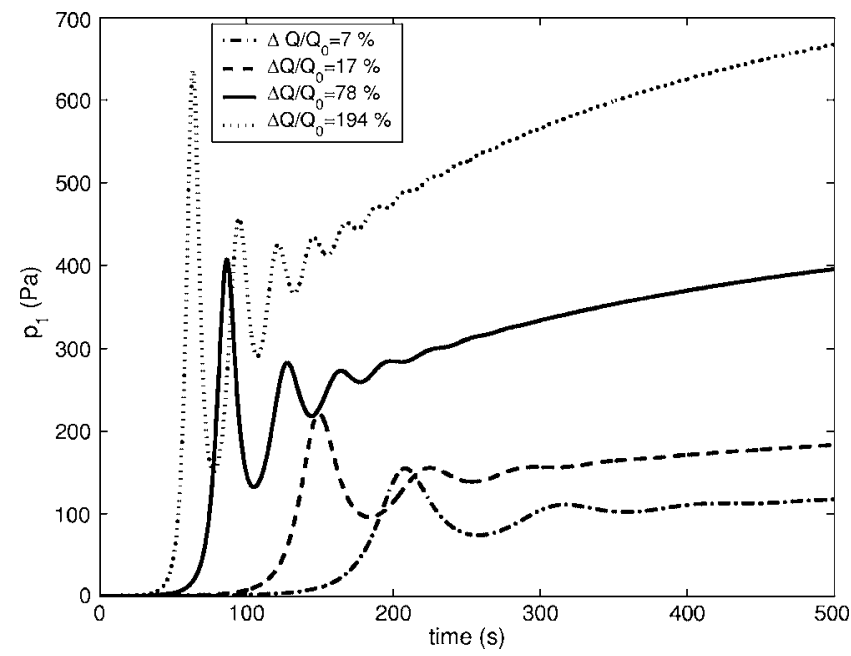

FIG. 6. Calculated evolution with time of acoustic pressure $p_{1}$ for various $\Delta Q$ increment of the external heating power supply above the critical value $Q_{0}$, when the nonlinear processes of higher harmonics generation and minor losses are neglected in the model. 



FIG. 7. Calculated evolution with time of acoustic pressure $p_{1}$ (top) and mean temperature gradient $\nabla T=\left[T_{s}(0, t)-T_{s}\left(-H_{s}, t\right)\right] / H_{s}$ along the stack (bottom) when $\Delta Q / Q_{0}=17 \%$.

that is when the processes of higher harmonics generation and minor losses are neglected in the model [this is done by setting $\tau_{\text {att }} \rightarrow \infty, \tau_{\text {minor }} \rightarrow \infty$, and $\beta_{\text {nl }}=0$ in Eq. (34)]. The comparative analysis of experimental and theoretical results depicted in Figs. 2 and 6 show that the model reproduces the experimentally observed dynamics quite well. From the quantitative point of view, both the durations from the beginning of the amplification to the stabilization of the acoustic wave and the pressure levels obtained in the stationary regime match experiments. From the qualitative point of view, the calculated curves draw an exponential growth and a first saturation, which are followed by a slow increase of the acoustic wave amplitude before its final stabilization for large $\Delta Q$ increments. The main difference between simulations and experiments concerns the observation of large low frequency damped oscillations of acoustic pressure amplitude in Fig. 6 before stabilization.

Figure 7 presents the evolution with time of the mean temperature gradient $\nabla T=\left[T_{s}(0, t)-T_{s}\left(-H_{s}, t\right)\right] / H_{s}$ along the stack which corresponds to one of the four regimes depicted in Fig. 6. Two observations may be made from the analysis of Fig. 7. First, the evolution with time of $\nabla T$ compared to $p_{1}(t)$ variations indicates that the large oscillations of acoustic pressure amplitude appear as a consequence of $\nabla T$ oscillations, which can be attributed to a "competition" between the external heating (tending to increase $\nabla T$ with subsequent thermoacoustic amplification) and the heat transfer due to acoustic streaming and acoustically enhanced conductivity (tending both to decrease $\nabla T$ ). Secondly, it is interesting to note in Fig. 7 that after the damping of oscillations, the mean temperature gradient $\nabla T$ decreases slowly whereas acoustic pressure is still increasing. This slow increase of acoustic pressure amplitude is actually controlled by the external heating (the characteristic time of amplitude stabilization of the acoustic wave being linked to the characteristic time of heat diffusion through the resonator walls) which does not necessarily lead to an increase of $\nabla T$ in the presence of high amplitude acoustic waves (as depicted in Fig. 7), but which leads to a change in the shape of the temperature field in such a way that the thermoacoustic amplification of sound is still occurring. The results depicted in Fig. 7 are in agreement with experimental observations and confirm that the saturation of the acoustic wave amplitude in thermoacoustic engines is a complex phenomenon, because the acoustically induced changes the temperature field in the thermoacoustic

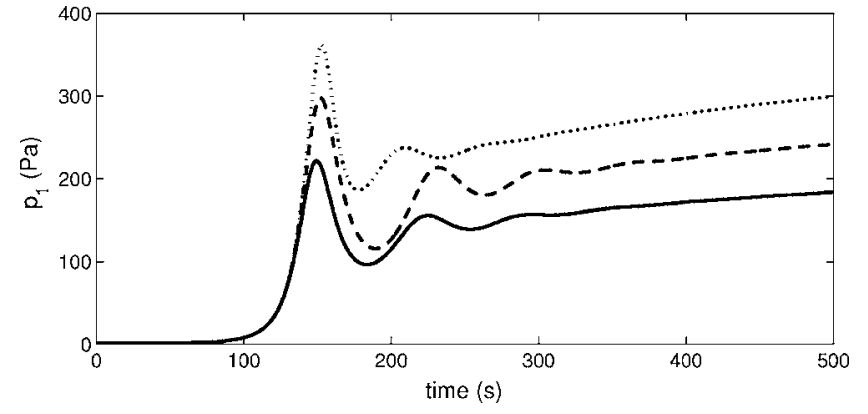

FIG. 8. Calculated evolution with time of acoustic pressure amplitude $p_{1}$ when $\Delta Q / Q_{0}=17 \%$ and when higher harmonics generation and minor losses are neglected. Solid line: $\left(\Gamma_{v} ; \Gamma_{\lambda}\right)=(4$ $\left.\times 10^{-8} ; 2 \times 10^{-6}\right)$. Dashed line: $\left(\Gamma_{v} ; \Gamma_{\lambda}\right)=\left(0 ; 2 \times 10^{-6}\right)$. Dotted line: $\left(\Gamma_{v} ; \Gamma_{\lambda}\right)=\left(4 \times 10^{-8} ; 0\right)$.

core involve changes in the spatial distribution of acoustic pressure, velocity and phase shift between pressure and velocity oscillations in the stack [3], with subsequent changes in the thermoacoustic amplification process.

It is also interesting to investigate the independent role of acoustic streaming and acoustically enhanced thermal conductivity on the dynamics of the wave amplitude growth. The results of such investigation are presented in Fig. 8 where the calculated evolution with time of acoustic pressure amplitude including both acoustic streaming and acoustically induced thermal conductivity (solid line) is compared first to the case when acoustic streaming is neglected (dashed line) and secondly to the case when acoustically induced thermal conductivity is neglected (dotted line). The results obtained demonstrate that both acoustic streaming and acoustically enhanced thermal conductivity contribute to the acoustic wave saturation, the most important contribution being that of acoustically enhanced conductivity (because the dashed curve in Fig. 8 is lower than the dotted curve).

In the following, all the nonlinear saturation processes described in Sec. III, including higher harmonics generation and minor losses, are taken into account in the simulations. Figure 9 presents the results obtained for the same $\Delta Q / Q_{0}$ increments as in Figs. 2 and 6. From the comparative analysis of Figs 2, 6, and 9, it can be concluded that the effect of minor losses and higher harmonics generation is not negligible because it provides an additional damping of the low frequency oscillations of acoustic pressure observed in Fig. 6 , in such a way that a very close agreement between theoretical results and experimental results of Fig 2 is finally obtained. Thus, this means that all the above mentioned nonlinear effects must be included in the model to obtain results which are qualitatively and quantitatively in agreement with experimental results of Fig. 2. Anyway, the previous interpretation of the role of acoustic streaming and acoustically enhanced thermal conductivity on the dynamic behavior of prime mover operation remains valid here.

\section{CONCLUSION}

In this paper, an experimental and theoretical investigation aiming at characterizing the nonlinear processes leading 


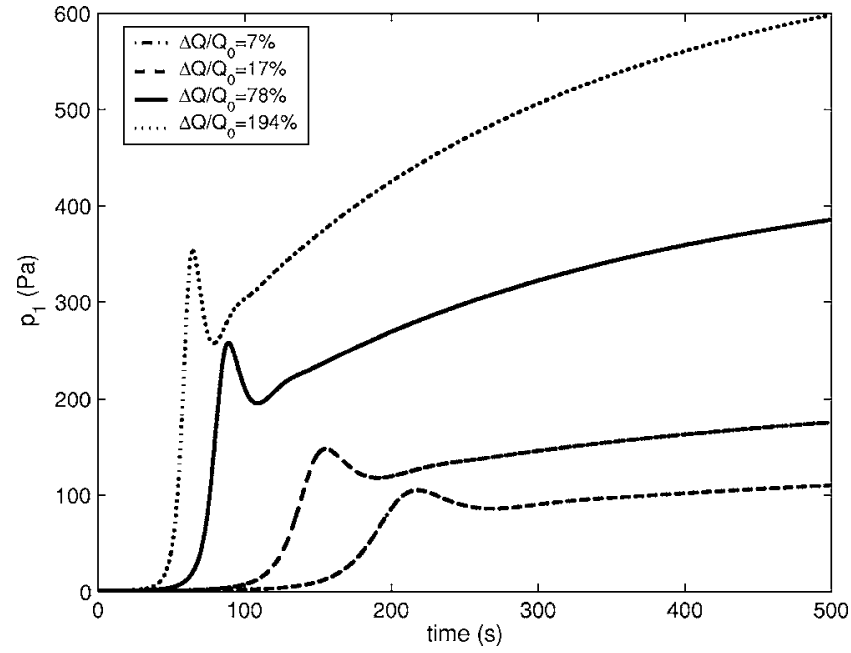

FIG. 9. Calculated evolution with time of acoustic pressure amplitude $p_{1}$ for various $\Delta Q$ increment of the external heating power supply above the critical value $Q_{0}$, when higher harmonics generation and minor losses are included in the model.

to steady-state sound in annular thermoacoustic prime movers is presented. From the analysis of the experimental results, it is demonstrated that the effects involving the reverse influence of the acoustic field on the temperature field need to be included in an adequate theory when trying to predict the amplitude of acoustic pressure in the stationary regime. Then, the simplified analytical description of the interactions between acoustic and thermal fields is proposed. The model is based on a one dimensional approach to describe heat transfer in the thermoacoustic core. It includes both the thermoacoustic heat pumping effect from hot to cold end of the stack (equivalent to acoustically enhanced thermal conductivity) and the forced convection due to the excitation of a mean unidirectional acoustic streaming. Furthermore, simplified descriptions of the additional saturation processes due to minor losses and higher harmonics generation are included in the model. The obtained equations are then solved using classical finite difference schemes, and the simulation results are compared with experiments: in spite of numerous approximations in the model, the simulation results reproduce remarkably the dynamical behaviors observed in experiments. Furthermore, the results give a new physical insight on the processes which control the saturation of the acoustic wave: it is demonstrated that the acoustically induced reduction of the imposed temperature gradient and the variations with time of the shape of the temperature field in the whole thermoacoustic core are predominantly responsible for the observed dynamics, but also that it is necessary to account for additional losses due to higher harmonics generation and minor losses when trying to reach quantitative agreement between simulations and experiments.

It should be recalled that, in the research field on thermoacoustics where the preliminary experimental analysis of fundamental physical processes remains essential, it is well established now that the saturation processes involved in a particular thermoacoustic device depend critically on the characteristics of the device itself. For instance, in the annular thermoacoustic prime mover which is studied in this pa- per, it has been demonstrated that both the dynamics of the wave amplitude growth and the acoustic levels obtained in the stationary regime depend on the stack heating process (e.g., the "internal" heating give rise to a periodic switch on-off of the thermoacoustic instability [26]) or on the orientation of the device relative to the gravity field (i.e., the effect of natural convection [27]). This renders the problem of predicting the performance of thermoacoustic device very difficult to solve, especially because the design of the existing thermoacoustic devices devoted to industrial applications is more and more complex. However, the analytical methods presented in this paper may be useful in the future as an interesting tool for engineers aiming at finding new ideas to optimize the performances of thermoacoustic devices.

\section{APPENDIX: ESTIMATES FOR HEAT TRANSFER PHENOMENOLOGICAL COEFFICIENTS}

In the one-dimensional approach presented in Sec. III B, the thermoacoustic core is composed of three different media [subscripted as $r, s$, and $w$ in Eqs. (3)-(5)], and it is assumed that the coupling between two different media is proportional to the difference between the temperatures of each medium. In the present case, it is not possible to have an analytical expression of the heat transfer coefficients $h^{r \leftrightarrow w}, h^{r \leftrightarrow s}$, and $h^{r \leftrightarrow \infty}$ which account both for the particular radial distribution of the acoustic streaming velocity and for the presence of a high amplitude acoustic wave [28,29]. So, the approximate values of $h$ are obtained here from known solutions giving an expression for the Nusselt number

$$
N u_{D}=\frac{h D}{\lambda}
$$

associated to more classical problems of heat transfer [20], where $\lambda$ stands for the thermal conductivity of fluid and $D$ is a characteristic dimension of the medium.

\section{Internal convection inside the resonator}

The main approximation here in the estimate of $h^{r \leftrightarrow w}$ consists in assuming that the unidirectional flow associated to the mean acoustic streaming is a laminar flow. From Ref. [30], the Nusselt number associated to internal forced convection in an horizontal tube (length $L$, diameter $D$ ) is given by the relationship

$$
N u_{D}=\left(3.66^{3}+1.66^{3} \sigma \operatorname{Re} \frac{D}{L}\right)^{1 / 3},
$$

which is valid if the conditions $\operatorname{Re}<900$ and $10^{-1}$ $<\sigma \operatorname{Re} D / L<10^{4}$ are satisfied, where $\sigma$ and $\operatorname{Re}$ denote the Prandtl number of the fluid and the Reynolds number associated to the streaming induced mean mass flow, respectively. In the present case (with $L \equiv H_{W}, D \equiv 2 r_{i}$ ), the approximate analytical expression for $h^{r \leftrightarrow w}$ is finally derived from Eqs. (A1) and (A2):

$$
h^{r \leftrightarrow w}=\frac{\lambda_{a}}{2 r_{i}}\left(3.66^{3}+1.66^{3} \sigma \frac{v_{w} 4 r_{i}^{2}}{\nu_{a} H_{W}}\right)^{1 / 3} .
$$




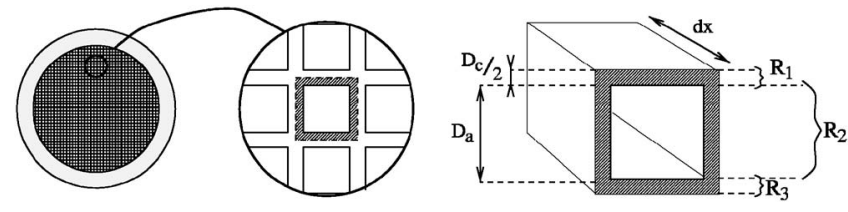

FIG. 10. Representation of the geometrical structure of the stack.

\section{Radial conduction and internal convection in the stack}

The conductive and convective radial heat transfer between the stack and the resonator wall can be estimated calculating the equivalent thermal resistance in the radial direction.

First, it is assumed that the actual conductive heat transfer $\Psi$ between stack and resonator walls is close to the conductive heat transfer through a cylindrical socket which is constituted of "half a thickness stack" in the radial direction from $r=r_{i} / 2$ to $r_{i}$ and of "half a thickness stainless steel" from to $r=r_{i}$ to $r_{i}+\left(r_{e}-r_{i}\right) / 2$, assuming moreover that internal and external surfaces are isothermal at temperatures $T_{s}$ and $T_{r}$, respectively. Consequently, this heat flux $\Psi$ is derived from the radial thermal resistance $R_{\text {th }}$ of the cylindrical socket as follows:

$$
\Psi=\frac{T_{r}-T_{s}}{R_{\mathrm{th}}},
$$

with

$$
R_{\mathrm{th}}=\frac{\ln \left(\frac{r_{i}}{r_{i} / 2}\right)}{2 \pi \lambda_{s}^{\perp} H_{s}}+\frac{\ln \left(\frac{r_{i}+\left(r_{e}-r_{i}\right) / 2}{r_{i}}\right)}{2 \pi \lambda_{i} H_{s}},
$$

where $\lambda_{s}^{\perp}$ denotes equivalent thermal conductivity of the stack in the radial direction.

Secondly, the effect of forced convection in the stack pores is included in the estimate of $\lambda_{s}^{\perp}$ in Eq. (A5), by calculating the thermal resistance of one stack pore in the radial direction. Figure 10 presents the geometrical structure of the stack with square channels (sidelength $D_{a}$ ) surrounded by ceramic walls (thickness $D_{c}$ ). The thermal resistance of one stack pore (length $H_{S}$ ) in the radial direction is written as the sum of three elementary resistances $R_{s}^{\perp}=R_{1}+R_{2}+R_{3}$, where

$$
R_{1}=R_{3}=\frac{D_{c} / 2}{\lambda_{c}\left(D_{a}+D_{c}\right) H_{s}}
$$

are the resistances to heat conduction in the ceramic walls (see Fig. 10), and where the resistance

$$
R_{2}=R_{c} / / R_{a}=\frac{R_{c} R_{a}}{R_{c}+R_{a}}
$$

is the parallel association of

$$
R_{c}=\left(\frac{D_{a}}{\lambda_{c} \frac{D_{c}}{2} H_{s}}\right) / /\left(\frac{D_{a}}{\lambda_{c} \frac{D_{c}}{2} H_{s}}\right)=\frac{D_{a}}{\lambda_{c} D_{c} H_{s}},
$$

which characterizes heat conduction in the ceramic walls, and of

$$
R_{a}=\left(h_{\mathrm{conv}} 4 D_{a} H_{s}\right)^{-1},
$$

which characterizes the forced internal convection in the channel ( "wet" surface $=4 D_{a} H_{s}$ ). In Eq. (A9), the heat transfer coefficient $h_{\text {conv }}$ is obtained from Eq. (A2):

$$
h_{\text {conv }}=\frac{\lambda_{a}}{D_{a}}\left(3.66^{3}+1.66^{3} \sigma \frac{\left(v_{w} / \Phi\right) D_{a}^{2}}{\nu_{a} H_{S}}\right)^{1 / 3} .
$$

Finally, reporting the expression $\lambda_{s}^{\perp}=\left(R_{s}^{\perp} H_{s}\right)^{-1}$ in Eq. (A5), the expression of the global conductive and convective heat flux between the stack and the resonator walls

$$
\frac{\Psi}{2 \pi r_{i} H_{s}}=h^{r \leftrightarrow s}\left(T_{r}-T_{s}\right)
$$

yields the expression of the global heat transfer coefficient

$$
h^{r \leftrightarrow s}=\frac{1}{2 \pi r_{i} H_{s}} \frac{1}{R_{\mathrm{th}}} .
$$

\section{External heat leakage}

Due to natural convection and thermal radiation, part of the thermal energy introduced in the resonator walls is transferred towards the external medium (at temperature $T_{\infty}$ ). It is assumed here in the estimate of the corresponding heat transfer coefficient $h^{r \leftrightarrow \infty}$ that the heat leaks due to natural convection prevail over those due to thermal radiation. Then from Ref. [20], the Nusselt number associated to external natural convection around an horizontal cylinder (diameter $D$, $30 \mu \mathrm{m}<D<10 \mathrm{~cm}$ ) at temperature $T_{r}$ is given by

$$
\sigma<10^{3}, N u_{D}=0.53\left(\frac{g \beta D^{3}\left(T_{r}-T_{\infty}\right)}{\nu \lambda}\right)^{1 / 4},
$$

where $\beta, \nu$, and $\lambda$ denote thermal expansion coefficient, kinematic viscosity and thermal conductivity of fluid, respectively, and where $g=9.81 \mathrm{~m} / \mathrm{s}^{-2}$ is the acceleration of the gravity field. Finally, the heat transfer coefficient $h^{r \leftrightarrow \infty}$ is derived from Eq. (A13) as follows:

$$
h^{r \leftrightarrow \infty}=0.53 \frac{\lambda_{a}}{2 r_{e}}\left(\frac{\beta g\left(2 r_{e}\right)^{3}}{\nu_{a} \lambda_{a}}\right)^{1 / 4}\left(T_{r}-T_{\infty}\right)^{1 / 4} .
$$

[1] N. Rott, Adv. Appl. Mech. 20, 135 (1980).

[2] G. W. Swift, J. Acoust. Soc. Am. 84, 1145 (1988).

[3] G. Penelet, S. Job, V. Gusev, P. Lotton, and M. Bruneau, Acust. Acta Acust. 91, 567 (2005).
[4] A. Atchley, H. Bass, and T. Hofler, in Frontiers in Nonlinear Acoustics, edited by M. Hamilton and D. Blackstock (Elsevier Applied Science, London, 1990), p. 603.

[5] G. Swift, J. Acoust. Soc. Am. 92, 1551 (1992). 
[6] H. Yuan, S. Karpov, and A. Prosperetti, J. Acoust. Soc. Am. 102, 3497 (1997).

[7] V. Gusev, H. Baillet, P. Lotton, and M. Bruneau, Acust. Acta Acust. 86, 25 (2000).

[8] V. Khokhlova, O. Sapozhnikov, S. Kasheeva, P. Lotton, V. Gusev, S. Job, and M. Bruneau, Sov. Phys. Izvestia Russian Acad. Sci. 64, 2334 (2001).

[9] R. S. Wakeland and R. M. Keolian, J. Acoust. Soc. Am. 112, 1249 (2002).

[10] R. S. Wakeland and R. M. Keolian, J. Acoust. Soc. Am. 115, 2071 (2004).

[11] T. Yazaki, A. Tominiga, and Y. Narahara, J. Heat Transfer 105, 889 (1983).

[12] T. Yazaki and A. Tominiga, Proc. R. Soc. London, Ser. A 454, 2113 (1998).

[13] S. Job, V. Gusev, P. Lotton, and M. Bruneau, J. Acoust. Soc. Am. 113, 1892 (2003).

[14] Y. Ueda, T. Biwa, and U. Mizutani, J. Acoust. Soc. Am. 115, 1134 (2004).

[15] G. Penelet, E. Gaviot, V. Gusev, P. Lotton, and M. Bruneau, Cryogenics 42, 527 (2002).

[16] G. B. Chen and T. Jin, Cryogenics 39, 843 (1999).

[17] D. Gedeon, Cryocoolers 9, 385 (1997).

[18] V. Gusev, S. Job, H. Baillet, P. Lotton, and M. Bruneau, J.
Acoust. Soc. Am. 108, 934 (2000).

[19] M. Mironov, V. Gusev, S. Job, H. Baillet, P. Lotton, M. Bruneau, and P. Piatakov, J. Acoust. Soc. Am. 112, 441 (2002).

[20] W. H. McAdams, Heat Transmission, 3rd ed. (Mc Graw-Hill, New York, 1953).

[21] M. Amari, V. Gusev, and N. Joly, Acust. Acta Acust. 89, 1008 (2003).

[22] O. V. Rudenko and S. I. Soluyan, Theoretical Foundations on Nonlinear Acoustics (Consultant Bureau, New York and London, 1977)

[23] W. Chester, J. Fluid Mech. 18, 44 (1964).

[24] I. E. Idelchik, Handbook of Hydraulic Resistance, 2nd ed. (Harper and Row, New York, 1986).

[25] M. N. Ozisik, Finite Difference Methods in Heat Transfer (CRC Press, Boca Raton, 1994).

[26] G. Penelet, V. Gusev, P. Lotton, and M. Bruneau, in Proceedings of the 4th World Congress on Ultrasonics (2003), p. 1053.

[27] G. Penelet,Ph.D. thesis, Université du Maine, 2004, URL http://tel.ccsd.cnrs.fr.

[28] A. Gopinath and A. Mills, J. Heat Transfer 116, 47 (1994).

[29] P. Vainshtein, M. Fichman, and C. Gutfinger, Int. J. Heat Mass Transfer 38, 1893 (1995).

[30] J. Taine and J. Petit, Transferts Thermiques, Mécanique des Fluides Anisothermes, 2nd ed. (Dunod, Paris, 1998). 